\title{
Electrospun Gelatin/Sodium Bicarbonate and Poly(lactide-co- $\varepsilon$-caprolactone)/Sodium Bicarbonate Nanofibers as Drug Delivery Systems
}

\author{
Qingqing Sang a, Gareth R. Williams ${ }^{\text {b }}$, Huanling Wu ${ }^{\text {a }}$, Kailin Liu ${ }^{\text {a }}$, Heyu Li ${ }^{\text {a }}$, Li-Min Zhu ${ }^{\text {acc,* }}$ \\ ${ }^{a}$ College of Chemistry, Chemical Engineering and Biotechnology, Donghua University, Shanghai 201620, \\ China \\ ${ }^{\mathrm{b}}$ UCL School of Pharmacy, University College London, 29-39 Brunswick Square, London, WC1N 1AX, \\ UK \\ ${ }^{\mathrm{c}}$ Key Laboratory of Science \& Technology of Eco-Textiles, Ministry of Education, Donghua University, \\ Shanghai 201620, China
}

\section{* Corresponding author:}

Prof. Li-Min Zhu

Tel: +86-21-62372655

E-mail:1zhu@dhu.edu.cn

\begin{abstract}
In this work, we report electrospun nanofibers made of model hydrophobic (poly(lactide-co- $\varepsilon$-caprolactone); PLCL) and hydrophilic (gelatin) polymers. We explored the effect of the incorporation of sodium bicarbonate (SB) into these fibres on drug release, using the potent antibacterial agent ciprofloxacin as a model drug. The fibers prepared are smooth and have relatively uniform diameters lying between $c a$. 600 and $900 \mathrm{~nm}$. The presence of ciprofloxacin in the fibers was confirmed using IR spectroscopy. X-ray diffraction showed the drug to be incorporated into the fibers in the amorphous form. In vitro drug release studies found that, as expected, more rapid drug release was seen with gelatin fibers than those made of PLCL, and a greater final release percentage was obtained. The inclusion of SB in the gelatin fibers imparts them with pH sensitivity: gelatin/SB fibers showed faster release at $\mathrm{pH} 5$ than $\mathrm{pH}$ 7, while fibers without SB gave the same release profiles at both pHs. The PLCL fibers have no $\mathrm{pH}$ sensitivity, even when SB was included, as a result of their hydrophobic structure precluding the ingress of solvent. In vitro cell culture studies showed that all the fibers are able to promote cell proliferation. The ciprofloxacin loaded fibers are effective in inhibiting Eschericha coli and Staphylococcus aureus growth in antibacterial tests. Thus, the gelatin-based fibers can be used as pH-responsive drug delivery systems, with potential applications for instance in the treatment of tumor resection sites. Should these become infected, the $\mathrm{pH}$ would drop, resulting in ciprofloxacin being released and the infection halted.
\end{abstract}

Keywords: electrospinning, drug delivery system, pH-responsive, anti-tumor, nanofiber

\section{Introduction}

Nanoscale fibers have been increasingly explored in recent years. There are many approaches available for 
preparing these, such as template polymerization, self-assembly, phase separation, and electrostatic spinning. The latter has attracted particular attention for the preparation of nanofiber materials because of its simplicity, low cost, possessing porous, high surface area to volume ratios and the wide variety of materials which can be processed [1, 2]. Nano- and micro-scale fibers find use in a range of areas including catalysis, environmental remediation, and for the delivery of active pharmaceutical ingredients [3]. Electrospinning has attracted particular attention for biomedical applications mainly due to growing interest in nano-technologies, and the attractive material properties which can be obtained (e.g. porosity, flexibility) [4]. For instance, researchers have explored the use of drugloaded electrospun fibers for the treatment or prevention of cancer. Ranganath et al. [5] prepared a electrospun poly(lactic-co-glycolic acid) nanofibers loaded with the anti-cancer drug paclitaxel, and found them to be effective against glioma tumors in vivo. In other work, Liu et al. [6] prepared electrospun fibers containing sodium dichloroacetate and found these to lead to the necrosis of tumor cells, resulting in a inhibition rate of up to $96 \%$. Tissue engineering, such as for the nerve, has also been widely explored [7].

An ideal drug delivery system (DDS) must be both safe and effective, and should also offer a specific delivery pattern. This might comprise site-specific drug delivery or extended release, for instance. Electrospun fibers have great promise in this regard. To achieve the desired release properties, the choice of polymer to be used requires careful consideration. A fast-dissolving polymer will give higher release than an insoluble material, but the solubility of the drug in the polymer is also important. In a study by Natu and co-workers [8], acetazolamide and timolol maleate-loaded fibers of poly( $\varepsilon$-caprolactone) and poly(oxyethylene-b-oxypropylene-boxyethylene) were prepared by blend electrospinning. Maleate was released faster than acetazolamide. High drug loadings, where crystals of the active ingredients were present, showed a greater burst release and faster release rate than low drug content fibers where the drug was spread evenly throughout the (slow-dissolving) polymer. Both the fiber composition and drug solubility in the polymer clearly influenced the release profiles seen.

Fibers can also be electrospun from stimuli-responsive materials, for instance those that change their properties with a change in temperature [9] or $\mathrm{pH}$. The latter is particularly important given the changes in $\mathrm{pH}$ which occur as a formulation taken orally passes through the human gastrointestinal tract, and differences between the physiological $\mathrm{pH}$ and that of the tumor microenvironment. A number of $\mathrm{pH}$-sensitive drug delivery systems generated by electrospinning have been reported. For example, Aguilar et al. [10] prepared composite mats of polyurethane and the $\mathrm{pH}$-sensitive polymer Eudragit ${ }^{\circledR}$ L100-55, loaded with paclitaxel by electrospinning. In vitro drug release, analyzed by high performance liquid chromatography, showed the composite mats give slow release of paclitaxel at $\mathrm{pH} 4$, and relatively rapid release at $\mathrm{pH}$ 6. Adopting a different approach, Zhao and coworkers [11] developed an acid-responsive nanofiber scaffold using electrospinning. The fibers comprised poly(L-lactic acid) loaded with sodium bicarbonate and ketoprofen. In acidic conditions, sodium bicarbonate reacts with protons to form $\mathrm{CO}_{2}$ gas, accelerating drug release. This reaction with acid can be used to generate pores in the fiber, and thus permit a drug cargo to diffuse easily into solution. In contrast, at neutral pH the SB is stable, and thus the fiber remains non-porous and impermeable. In this way, $\mathrm{pH}$ sensitivity can be achieved without the need for bespoke polymers, allowing a much wider range of responsive systems to be developed.

Our ability to treat and cure cancer has improved tremendously in recent years [12]. However, there are still numerous deaths from cancer every year, and thus the search for new and more effective therapies continues. Surgical removal of the tumor is perhaps the "gold standard" treatment, where the growth is accessible. This comes with potential problems though: for instance, the operation may cause an inflammatory reaction, which in 
turn can lead to the recurrence of the tumor [13]. This reaction is also associated with a lowering of the local tissue $\mathrm{pH}$, and thus a $\mathrm{pH}$-responsive system might be beneficial here [14].

In this study, we report electrospun fibers developed using sodium bicarbonate (SB) as a pH-responsive component. We explored the ability of SB to impart $\mathrm{pH}$ sensitivity on two polymers which do not normally possess this: gelatin and poly(lactide-co-e-caprolactone). These are respectively model hydrophilic and hydrophobic polymers, and this is the first time that the ability of sodium bicarbonate to induce pH-sensitivity in analogous nanofibers of differing hydrophilicity has been. The ultimate aim of this work is to develop novel antitumor therapeutics.

Gelatin is a hydrophilic natural biopolymer. It has been widely used in food, medicine and cosmetic applications as well as in biomaterials [15, 16], and is the major structural protein in animal skin and bones. Gelatin has very good biological compatibility, and thus is attractive as a potential drug carrier [17]. However, gelatin dissolves quickly into aqueous media (regardless of $\mathrm{pH}$ ), and thus although gelatin fibers can be prepared by electrospinning they undergo very rapid release unless some post-fabrication modification is performed. Approaches to ameliorate this issue include using a layer-by-layer approach to combine multiple polymers in a single system. For instance, Mandal et al. [18] prepared a silk fibroin/gelatin multilayer film and were able to control the release of the drug incorporated. Such a fabrication process is relatively complex though, and a simple option would be advantageous. Here, we opted to simply cross-link the gelatin molecules in the fibers after their fabrication, using glutaraldehyde as a crosslinking agent [19]. Zhang and co-workers have previously demonstrated the utility of this approach by employing a saturated glutaraldehyde vapor to improve the thermostability and mechanical properties of gelatin nanofibers [20].

Poly(lactide-co-e-caprolactone) (PLCL) is a hydrophobic aliphatic polyester copolymer. It has the advantages of good biocompatibility, a lack of toxicity in vivo, and high mechanical strength. It is also easy to process by electrospinning, and PLCL products have been explored in tissue engineering, and for wound dressings, drug delivery, and so forth [21, 22].

The model drug ciprofloxacin was loaded into both the PLCL and gelatin systems being explored. Ciprofloxacin is a third generation quinolone, with broad-spectrum antibacterial activity [23, 24]. By developing novel $\mathrm{pH}$-responsive nanofiber membranes containing ciprofloxacin, we hoped to develop scaffolds which ultimately might be used after tumour resection surgery. A detailed comparison of the drug release from hydrophobic PLCL and hydrophilic gelatin-based fibers is reported, and their potential applications studied.

\section{Experimental}

\subsection{Materials}

Gelatin (powder, purity > 99.5\%) was supplied by the Sinopharm Chemical Reagent Co., Ltd), while dimethyl sulfoxide (DMSO) was sourced from J\&K Chemicals. Dulbecco's Modified Eagle Medium (DMEM) was provided from Jinuo Biological Medicine Technology Ltd. Poly(lactide-co- $\varepsilon$-caprolactone) (PLCL, PCL:PLLA=1:1) came from Jinan Daigang Biomaterials. 1,1,1,3,3,3-Hexafluoroisopropanol (HFIP, purity > 95.5\%), ciprofloxacin (purity > 98\%), phosphate-buffered saline ( $\mathrm{PBS}$, sodium phosphate $=10 \mathrm{mM}, \mathrm{NaCl}=150$

$\mathrm{mM} ; \mathrm{pH}=7.4$ ), sodium bicarbonate ( $\mathrm{SB}$, powder, purity > 99.5\%), penicillin/streptomycin, thiazolyl blue tetrazolium bromide (MTT) were supplied by Sigma-Aldrich Ltd. L929 cells were purchased from the Chinese 
Institute of Biochemistry and Cell Biology. An acetic acid buffer (AA, sodium acetate=25 mM; pH=5.0) was prepared in-house. All other chemicals were at least analytical grade, and water was doubly distilled prior to use.

\subsection{Electrospinning}

Gelatin or PLCL were dissolved in hexafluoroisopropanol (HFIP) with SB, as detailed in Table 1. Ciprofloxacin was added into selected solutions at a drug to polymer ratio of 1:9 (w/w). The solutions were magnetically stirred in closed glass bottles sealed with parafilm at room temperature for $24 \mathrm{~h}$. They were then loaded into a $5 \mathrm{~mL}$ plastic syringes fitted with a stainless steel spinneret (internal diameter $0.5 \mathrm{~mm}$ ). A syringe pump (KDS100, Cole-Parmer) was used to drive the working fluids, at a flow rate of $0.8 \mathrm{~mL} / \mathrm{h}$. The electrospinning process was carried out with a high voltage power supply (ZGF-2000, Shanghai Sute Electrical Co. Ltd.) used to provide a potential difference of $15 \mathrm{kV}$ between the spinneret and collector plate. The latter had dimensions of $8 \times 9 \mathrm{~cm}$ and was covered in aluminum foil. The distance between the spinneret and aluminum foil was $23 \mathrm{~cm}$. Experiments were performed at 20-25 ${ }^{\circ} \mathrm{C}$, and $33-45 \%$ relative humidity. The electrospun products were dried in a vacuum chamber (DZF-6050, Shanghai Laboratory Instrument Work Co. Ltd.) for two days at $25^{\circ} \mathrm{C}$. After that, the gelatin fibers were crosslinked using an aqueous glutaraldehyde solution (25.0 $28.0 \%(\mathrm{v} / \mathrm{v}))$ to render them insoluble in water. The glutaraldehyde solution $(10 \mathrm{~mL})$ was placed at the bottom of a brown translucent desiccator, and the fiber samples placed in a Petri dish and mounted on a rack above this solution for approximately $8 \mathrm{~h}$. All further experiments were performed on cross-linked fibers in the case of gelatin.

Table 1. Details of the electrospinning solutions used in this study.

\begin{tabular}{ccccc}
\hline Fiber ID & Gelatin conc (w/v) & PLCL conc (w/v) & SB conc (w/v) & Ciprofloxacin conc (w/v) \\
\hline $\mathrm{G}^{+}$ & $15 \%$ & - & - & $10 \%$ \\
$\mathrm{G}^{-}$ & $15 \%$ & - & - & - \\
$\mathrm{GS}^{+}$ & $15 \%$ & - & $5 \%$ & $10 \%$ \\
$\mathrm{GS}^{-}$ & $15 \%$ & - & $5 \%$ & - \\
$\mathrm{P}^{+}$ & - & $5 \%$ & - & $10 \%$ \\
$\mathrm{P}^{-}$ & - & $5 \%$ & - & - \\
$\mathrm{PS}^{+}$ & - & $5 \%$ & $5 \%$ & $10 \%$ \\
$\mathrm{PS}^{-}$ & - & $5 \%$ & $5 \%$ & - \\
\hline
\end{tabular}

\subsection{Fiber morphology}

The morphological structure of the nanofibers was characterized by scanning electron microscopy (SEM; JSM-5600 LV microscope, JEOL) at a voltage of $10 \mathrm{kV}$. Sections of the fiber mats $(1 \times 0.5 \mathrm{~cm})$ were cut, and sputter coated with platinum for $c a .30 \mathrm{~s}$ before SEM was performed. The diameters of the fibers were then measured using the ImageJ software (National Institutes of Health): $c a .50$ fibers from each formulation were randomly selected for measurement. The formulations were also examined by transmission electron microscopy (TEM, H-800 instrument, Hitachi). Samples for TEM were directly electrospun onto copper grids.

\subsection{Additional fiber characterization}


Fourier transform infrared (FT-IR) spectra were obtained on a Nicolet-Nexus 670 FT-IR spectrometer (Nicolet Instrument Corporation) over the range $500-4000 \mathrm{~cm}^{-1}$ and with a resolution of $2 \mathrm{~cm}^{-1}$. X-Ray diffraction (XRD) patterns were collected over $5-60^{\circ}$ on a D/Max-BR diffractometer (Rigaku) supplied with $\mathrm{Cu}$ $\mathrm{K} \alpha$ radiation $(40 \mathrm{kV} / 30 \mathrm{~mA}, \lambda=1.5418 \AA)$.

Water contact angles were used to quantify the hydrophilic-hydrophobic properties of the fibers. 15 circular cover slips (diameter ca. $14 \mathrm{~mm}$ ) were placed on the aluminum foil covered collector plate, and fibers electrospun onto them for approximately $7 \mathrm{~h}$. After drying in a vacuum drying oven, the fiber coated cover slips were used to determine contact angles using a $322 \mathrm{~W}$ instrument (Thermo Cahn). A water droplet (ca. $2 \mu \mathrm{L})$ was placed onto the surface of the fibers at room temperature, and digital images recorded. At least three samples of each formulation were assessed, and three measurements made for each sample. Contact angles were quantified immediately after the water droplet had come into contact with the fibers.

\subsection{In vitro drug release}

$0.1 \mathrm{~g}$ of the ciprofloxacin-loaded fibers were placed in a centrifuge tube with $20 \mathrm{~mL}$ of PBS (pH 7.4) or AA ( $\mathrm{pH}$ 5.0) buffer solution. The centrifuge tubes were incubated in a constant temperature shaker $\left(37^{\circ} \mathrm{C}, 100 \mathrm{rpm}\right)$ for one week. During this time, $1.0 \mathrm{~mL}$ of the release medium was periodically removed and $1.0 \mathrm{~mL}$ of fresh pre-warmed PBS or AA solution added to keep the volume constant. The concentration of ciprofloxacin was quantified at $277 \mathrm{~nm}$ using a UV-vis spectrometer (UV-1800, SHJH Company). Experiments were undertaken in triplicate, and the results are reported as mean \pm S.D.

\subsection{Antibacterial activity}

The antibacterial activity of the fibers was determined using the zone of inhibition test. $10^{5}$ colony forming units exemplar Gram-negative (Eschericha coli; ATCC 29522) and Gram-positive (Staphylococcus aureus; ATCC 6538), were separately cultured on lauria broth. The pH of the liquid broth was adjusted to 6.8, then 100 $\mu \mathrm{L}$ aliquots of the bacterial suspensions were poured into Petri dishes $(9 \mathrm{~cm}$ in diameter). The dishes were rested for 10 minutes. Circular samples (diameter $c a .14 \mathrm{~mm}$ ) cut from the fiber mat were added to the plates. Incubation then proceeded for three days at $37{ }^{\circ} \mathrm{C}$, after which inhibition zones were measured. Experiments were undertaken in triplicate, and the results are reported as mean \pm S.D.

\subsection{In vitro cytotoxicity experiments}

The in vitro cytotoxicity of the fiber membranes was tested using the MTT (thiazolyl blue tetrazolium bromide) assay. L929 fibroblast cells were cultivated in complete DMEM (DMEM supplemented with 10\% (v/v) inactivated fetal bovine serum, and $1 \%(\mathrm{v} / \mathrm{v})$ penicillin-streptomycin solution $(100 \mathrm{units} / \mathrm{mL}))$ at $37^{\circ} \mathrm{C}$. Fibercoated circular cover slips (prepared as for the contact angle experiments) were placed in the wells of a 24-well plate. The plates were sterilized under UV irradiation for $2 \mathrm{~h}$, then in alcohol steam for $6 \mathrm{~h}$. Before use, they were washed with PBS three times to remove any residual alcohol. Each well was augmented with $400 \mu \mathrm{L}$ of complete DMEM and incubated $\left(5 \% \mathrm{CO}_{2}, 37^{\circ} \mathrm{C}\right)$ for ca. $2 \mathrm{~h}$.

The L929 cells in culture underwent trypsinization to remove them from the culture flask, after which they were counted and resuspended at $2 \times 10^{4}$ cells $/ \mathrm{mL}$. $200 \mu \mathrm{L}$ of these dissociated L929 cells were added to each well of the plate, which was incubated $\left(37^{\circ} \mathrm{C}, 5 \% \mathrm{CO}_{2}\right)$ for 1,3 or 5 days. After incubation, the DMEM solution 
was removed, the wells washed twice with PBS, and $40 \mu \mathrm{L}$ of the MTT reagent solution $(0.5 \%(w / v)$ in PBS) and $360 \mu \mathrm{L}$ of DMEM added. The plates were incubated $\left(37^{\circ} \mathrm{C}, 5 \% \mathrm{CO}_{2}\right)$ for approximately $4 \mathrm{~h}$, after which the fluid in the wells was aspirated and replaced with $400 \mu \mathrm{L}$ of DMSO. The plates were placed on a shaker for 30 min, then transferred into a 96 well plate. The optical density at $570 \mathrm{~nm}$ was quantified with a microplate reader (Multiskan, Thermo-Fisher). Three independent experiments were performed, with triplicates in each.

\section{Results and discussion}

\subsection{Fiber morphology}

The morphology of all the fibers was studied by SEM and TEM. The images obtained are shown in Fig. 1 and Fig. S1 (Supporting Information). SEM reveals that all the fibers are cylindrical in shape, and their surfaces are uniform and largely smooth. The gelatin $\left(\mathrm{G}^{-}\right)$and PLCL $\left(\mathrm{P}^{-}\right)$fiber diameters are $662 \pm 121 \mathrm{~nm}$ and $803 \pm 157$ $\mathrm{nm}$, respectively, while the gelatin/SB $\left(\mathrm{GS}^{-}\right)$and PLCL/SB $\left(\mathrm{PS}^{-}\right)$are essentially the same at $637 \pm 146 \mathrm{~nm}$ and $844 \pm 122 \mathrm{~nm}$. The presence of SB thus has no marked effect on diameter. The drug-loaded $\mathrm{G}^{+}$and $\mathrm{P}^{+}$analogues have diameters somewhat smaller than the drug-free systems: $603 \pm 128 \mathrm{~nm}$ and $732 \pm 130 \mathrm{~nm}$ (see Fig. S1). This is also true for materials made up of gelatin/SB/ciprofloxacin $\left(\mathrm{GS}^{+}\right)$and PLCL/SB/ciprofloxacin $\left(\mathrm{PS}^{+}\right)$, which have diameters of $625 \pm 125 \mathrm{~nm}$ and $798 \pm 123 \mathrm{~nm}$ (see Fig. 1). This is expected to be because the addition of ciprofloxacin increases the conductivity of the electrospinning solution.

Fig. 1(e) and (f) show the SEM images of $\mathrm{GS}^{-}$and $\mathrm{GS}^{+}$after cross-linking. The fibers' morphology has barely changed, but their diameters have become larger. The same effect is seen for the SB-free systems $\mathrm{G}^{-}$ and $\mathrm{G}^{+}$(Fig. S1), and can possibly be attributed to the formation of aldimine linkages $(\mathrm{CH}=\mathrm{N})$. TEM images (Fig. 1(g)-(j)) show the fibers to comprise monolithic structures, with no core/shell structure, as would be expected from a single fluid electrospinning experiment. 

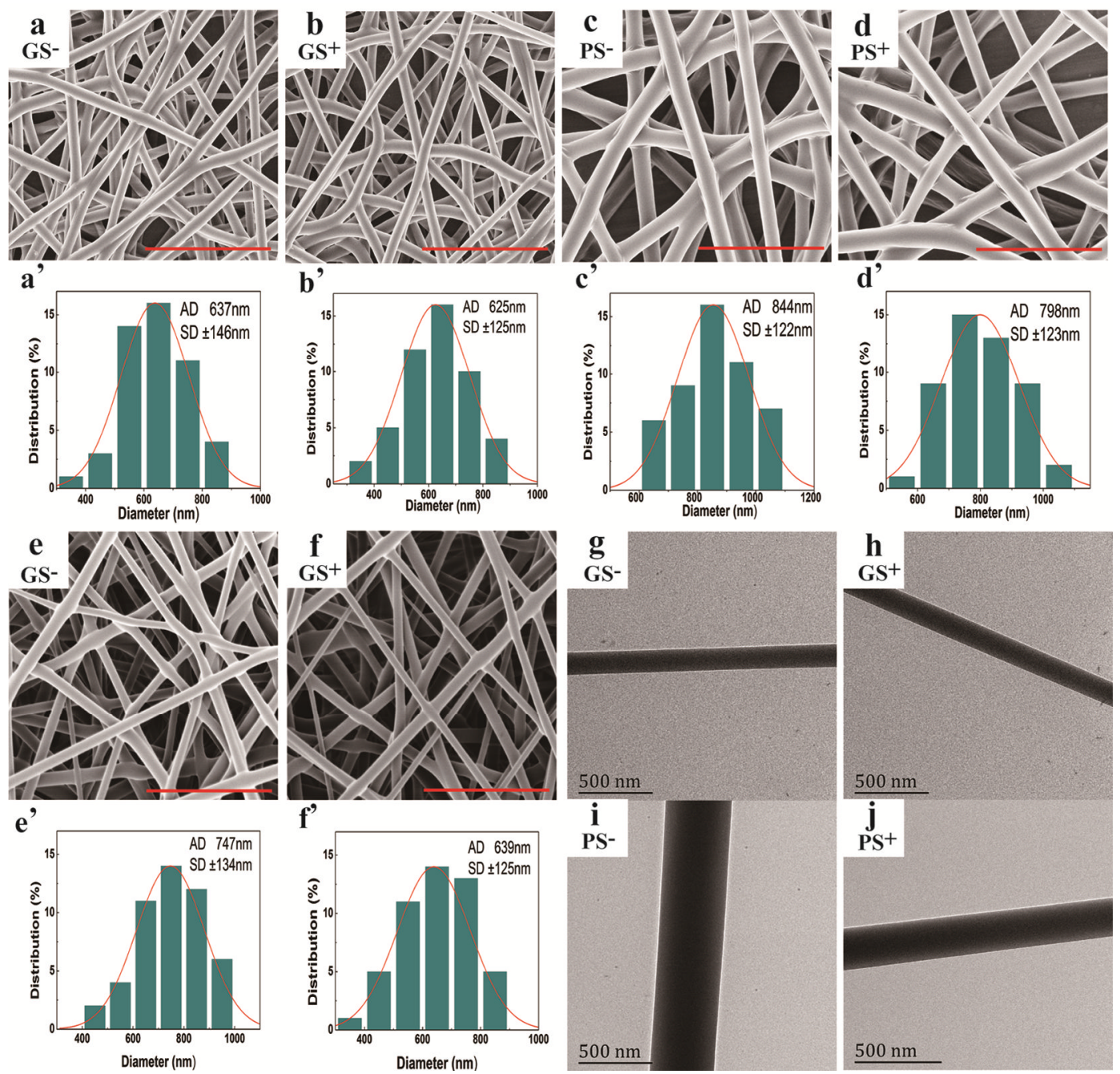

Fig. 1. SEM and TEM images of the fibers generated. SEM images are given for (a) GS ; (b) $\mathrm{GS}^{+}$; (c) $\mathrm{PS}^{-}$; (d) $\mathrm{PS}^{+}$; (e) GS ${ }^{-}$after cross-linking; and (f) $\mathrm{GS}^{+}$after cross-linking, together with the fiber diameter distributions (a'f'). TEM images are presented for: (g) GS ; (h) $\mathrm{GS}^{+}$; (i) $\mathrm{PS}^{-}$; and (j) $\mathrm{PS}^{+}$. The scale bar in the SEM images represents $10 \mu \mathrm{m}$.

\subsection{Further characterization}

The XRD patterns and FT-IR spectra of gelatin, PLCL, ciprofloxacin and the different fibers are presented in Fig. 2. The XRD data in Fig 2(a) show that PLCL is semi-crystalline, with two Bragg reflections. The gelatin XRD pattern confirms it to be an amorphous material, with no reflections visible and only the broad humps typical of amorphous systems. Ciprofloxacin exhibits numerous reflections in its XRD pattern, and thus it is clearly a crystalline material [25, 26]. The PLCL formulations have the peaks of the polymer in their XRD patterns, but no additional features can be seen. The gelatin fibers show no Bragg reflections in their patterns, only broad humps. Thus, the ciprofloxacin is incorporated into the fibers in the amorphous physical form by dint of the very rapid nature of the electrospinning process [27].

FT-IR spectra are given in Fig 2(b). Gelatin shows peaks at $3293 \mathrm{~cm}^{-1}$ (H-bonded -OH stretching), $1658 \mathrm{~cm}^{-}$ ${ }^{1}$ ( $\mathrm{C}=\mathrm{O}$ stretching; amide I band) and $1538 \mathrm{~cm}^{-1}$ (bending of $\mathrm{N}-\mathrm{H}$ and stretching of $\mathrm{C}-\mathrm{N}$; amide II) [28]. PLCL 
has key vibrations at $1760 \mathrm{~cm}^{-1}$ which arise from ester bonds $(-\mathrm{C}(=\mathrm{O}) \mathrm{O}-)$, and at $2940 \mathrm{~cm}^{-1}$ from methylene ($\mathrm{CH}_{2}$ ) groups $[29,30]$. The spectrum of ciprofloxacin displays characteristic peaks at $1599 \mathrm{~cm}^{-1}$ and $1499 \mathrm{~cm}^{-1}$, corresponding to benzene ring stretching vibrations, carboxylate stretching at $1620 \mathrm{~cm}^{-1}$, and bands at $1090 \mathrm{~cm}^{-1}$ from the stretching of C-F, and at $3049 \mathrm{~cm}^{-1}$ from O-H stretching [31]. The spectra of the drug-free $\mathrm{GS}^{-}$and $\mathrm{PS}^{-}$ fibers show the same features as the raw polymer. $\mathrm{GS}^{+}$and $\mathrm{PS}^{+}$have very similar spectra to the pure polymer, but with an additional peak at $1090 \mathrm{~cm}^{-1}$ and (in the case of $\mathrm{PS}^{+}$) $1620 \mathrm{~cm}^{-1}$ indicating the successful incorporation of ciprofloxacin into gelatin or PLCL, respectively.
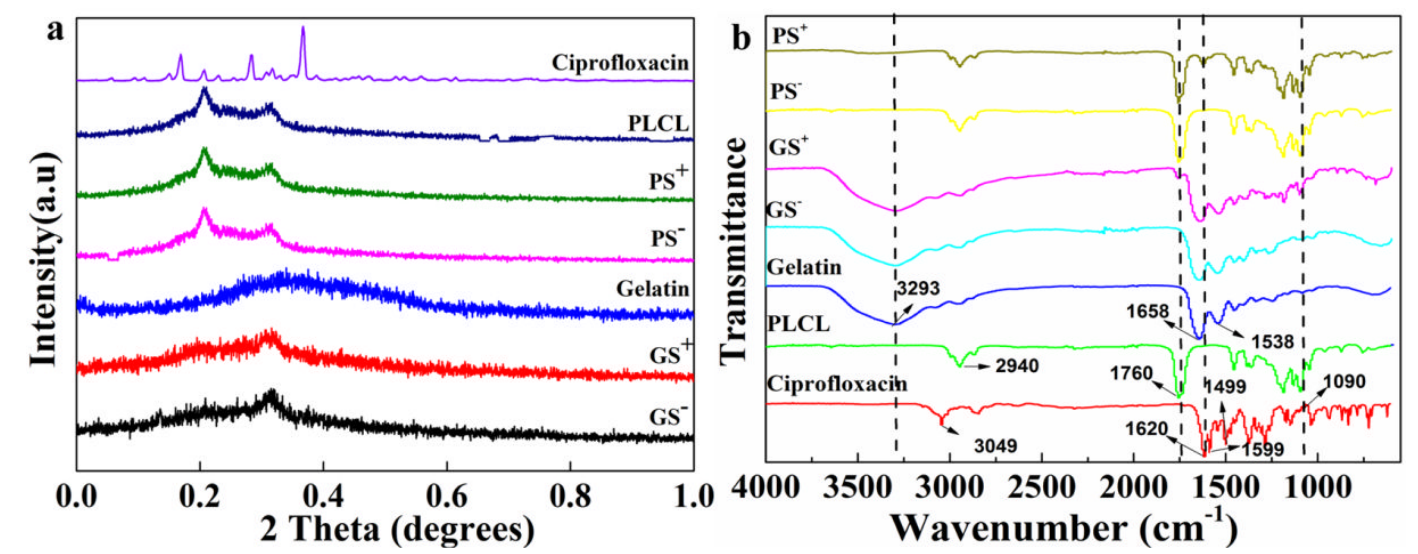

Fig. 2. (a) XRD patterns and (b) FT-IR spectra of gelatin, PLCL, ciprofloxacin and selected fibers.

Contact-angle data and images obtained immediately after addition of the water droplet to the fiber surface are presented in Fig. 3 and Table 2. As described in the literature [32, 33], the gelatin nanofiber membranes have hydrophilic surfaces, with contact angles below $40^{\circ}$. In contrast, the PLCL fibers are hydrophobic and have high contact angles $>100^{\circ}$. The addition of the hydrophilic ciprofloxacin reduced the contact angle for both the gelatin and PLCL fibers. The contact angles also decrease slightly with the addition of SB.
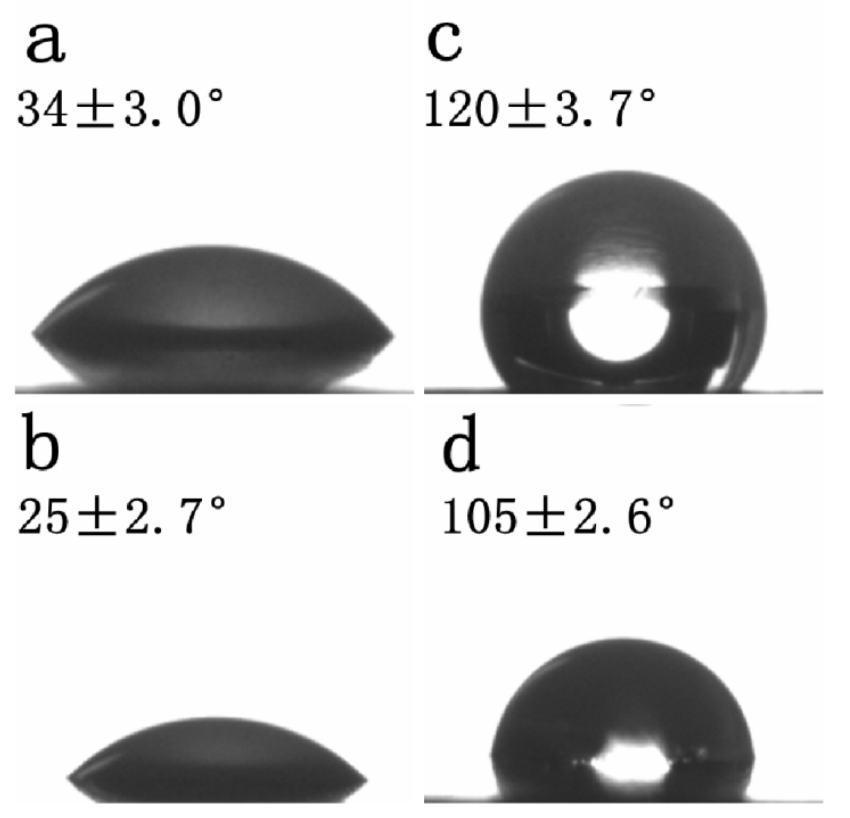

Fig. 3. Water contact angles of gelatin and PLCL nanofiber membranes: (a) GS ; (b) GS ${ }^{+}$; (c) $\mathrm{PS}^{-}$; (d) $\mathrm{PS}^{+}$. 
Table 2. The contact angles of the fibers.

\begin{tabular}{cccc}
\hline Sample & Contact-angle & Sample & Contact-angle \\
\hline $\mathrm{GS}^{-}$ & $34 \pm 3.0^{\circ}$ & $\mathrm{G}^{-}$ & $33 \pm 3.1^{\circ}$ \\
$\mathrm{GS}^{+}$ & $25 \pm 2.7^{\circ}$ & $\mathrm{G}^{+}$ & $21 \pm 2.3^{\circ}$ \\
$\mathrm{PS}^{-}$ & $120 \pm 3.7^{\circ}$ & $\mathrm{P}^{-}$ & $119 \pm 4.8^{\circ}$ \\
$\mathrm{PS}^{+}$ & $105 \pm 2.6^{\circ}$ & $\mathrm{P}^{+}$ & $110 \pm 5.2^{\circ}$ \\
\hline
\end{tabular}

\subsection{In vitro ciprofloxacin release and antibacterial activity}

Fig. 4 and Fig. S2 illustrate the in vitro ciprofloxacin release profiles in PBS (pH 7.4) or AA (pH 5.0) at 37 ${ }^{\circ} \mathrm{C}$. The $\mathrm{SB}$-free $\mathrm{G}^{+}$fibers and $\mathrm{P}^{+}$fibers have very similar release profiles in AA and PBS, with no $\mathrm{pH}$ sensitivity (Fig. S2), with a burst release of around $15 \%$ at the start of the experiment, and then sustained release over $30-$ $50 \mathrm{~h}$. The $\mathrm{GS}^{+}$fibers exhibit a burst of release in the first $6 \mathrm{~h}$, and the total amounts of ciprofloxacin released reached $79.8 \%$ and $58.5 \%$ after incubation for $29 \mathrm{~h}$ in AA and PBS, respectively. There is clear $\mathrm{pH}$ sensitivity visible in the release profiles. The $\mathrm{PS}^{+}$fibers also show a burst release, but this is much reduced over that noted with $\mathrm{GS}^{+}$. The total release amounts reached $46.1 \%$ and $46.9 \%$ after incubation for $45 \mathrm{~h}$ in AA and PBS, with the release profiles in both conditions being essentially superimposable. The total amount of ciprofloxacin released from the gelatin fibers is higher than that from the PLCL fibers, which is believed to be because ciprofloxacin releases more easily from a hydrophilic material than a hydrophobic material [34].

The SB-containing gelatin fibers showed clear $\mathrm{pH}$ sensitivity, with acidic conditions promoting ciprofloxacin release. This is expected to be because of the release of $\mathrm{CO}_{2}$ gas when $\mathrm{SB}$ reacts with protons, therefore creating channels inside the fibers, forming a porous structure, and promoting more ciprofloxacin release. SEM images were collected to assess this hypothesis, and are shown in Fig. 5. Many particles, believed to be drug [35], can be seen on the surface of the $\mathrm{GS}^{+}$fibers, particularly after immersion in the AA buffer solution. This confirms the reaction of SB from within the fibers with protons in the release milieu, with this effect being much greater with the acidic AA buffer. However, the PLCL fibers show very few solid particles on their surfaces. We presume this is because the hydrophobic nature of the fibers makes it very difficult for the buffer solution to penetrate into the fibers, and thus the SB has little opportunity to react and generate $\mathrm{CO}_{2}$. Clearly, the inclusion of SB alone is not sufficient to impart pH sensitivity, and the properties of the polymer are also crucial here. The $\mathrm{pH}$-sensitive properties of the $\mathrm{GS}^{+}$fibers might make them suitable to be implanted after tumor resection to help prevent infection and the recurrence of cancer. 


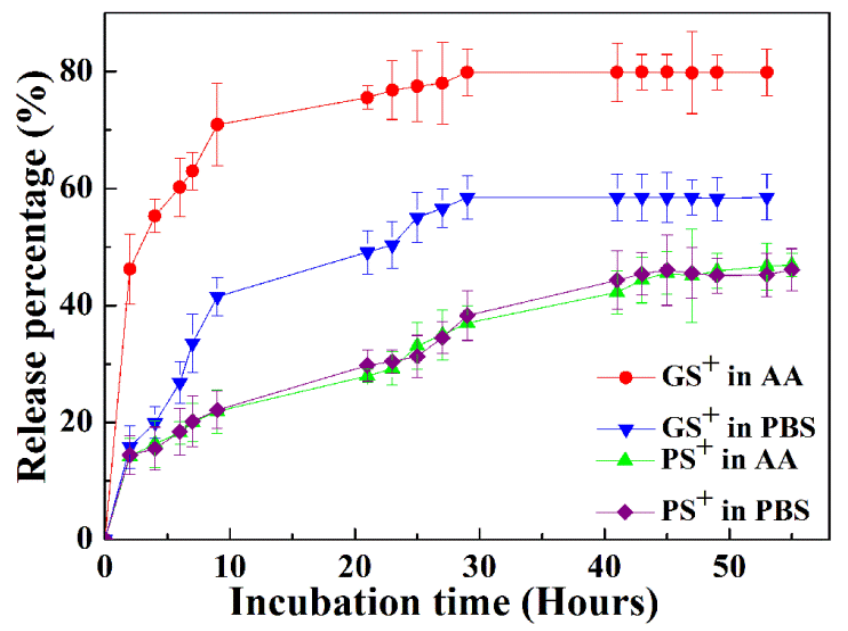

Fig. 4. In vitro ciprofloxacin release profiles from $\mathrm{GS}^{+}$and $\mathrm{PS}^{+}$fibers in PBS (pH 7.4) or AA (pH 5.0) buffer solution.

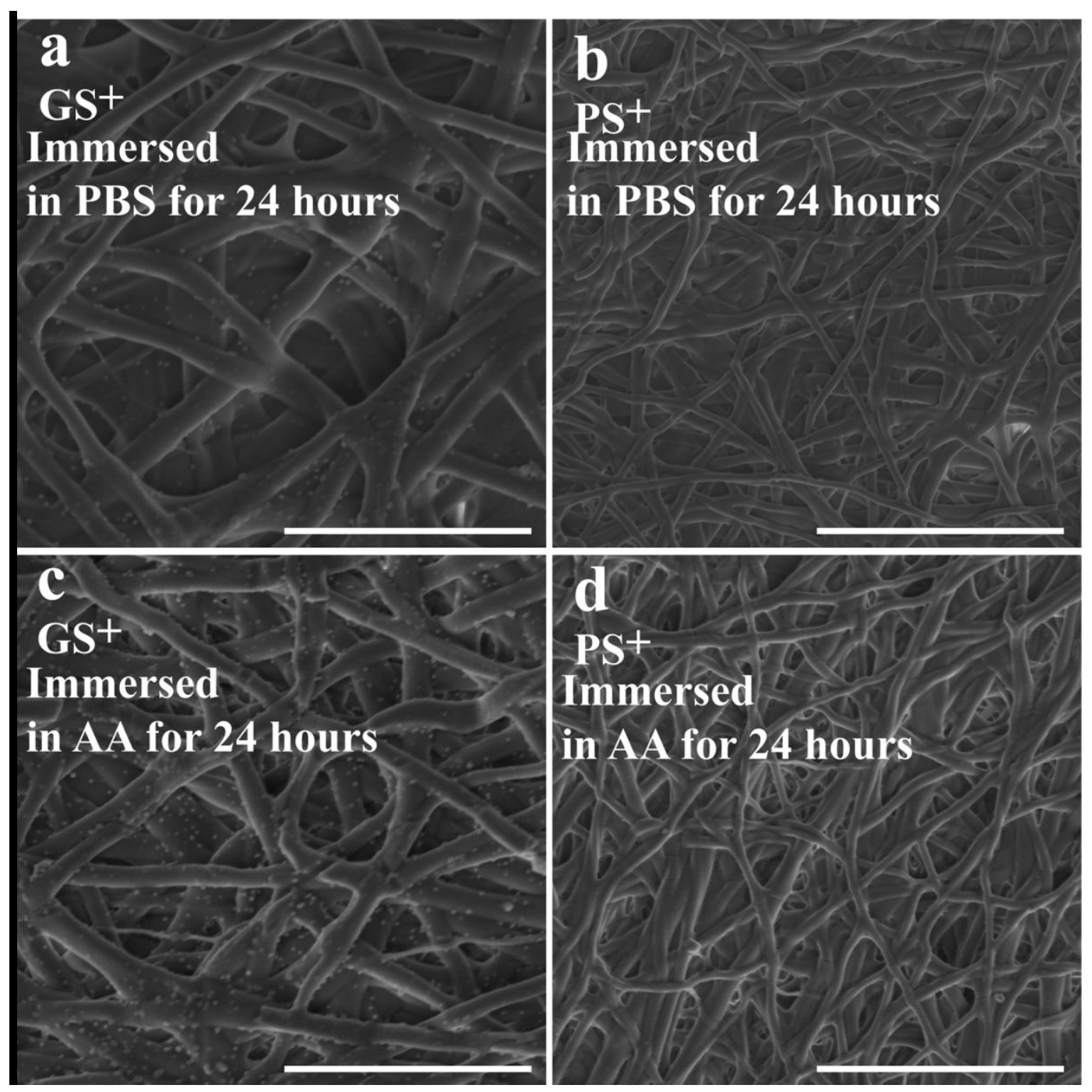

Fig. 5. SEM images of $\mathrm{GS}^{+}$and $\mathrm{PS}^{+}$after immersion in PBS (pH 7.4) ((a), (b)) or AA (pH 5.0) ((c), (d)) for 24 hours. Scale bar: $10 \mu \mathrm{m}$. 
Bacterial inhibition tests were used to evaluate the antibacterial activity of the ciprofloxacin released from the fibers against Eschericha coli and Staphylococcus aureus. The results can be found in Fig. 6 and Table 3. Clearly, the drug-free fibers have no antibacterial efficacy. In contrast, both the ciprofloxacin-containing fibers showed antibacterial activity, with inhibition zones of $26-42 \mathrm{~mm}$. The zone with $\mathrm{GS}^{+}$was larger than that with $\mathrm{PS}^{+}$. This is expected to be because a greater percentage of the drug is free from the gelatin fibers, as revealed from the in vitro drug release experiments. The addition of SB also resulted in an increased diameter of the inhibitory zone. The inhibition zones against Staphylococcus aureus are larger than with Eschericha coli, consistent with the literature [36].

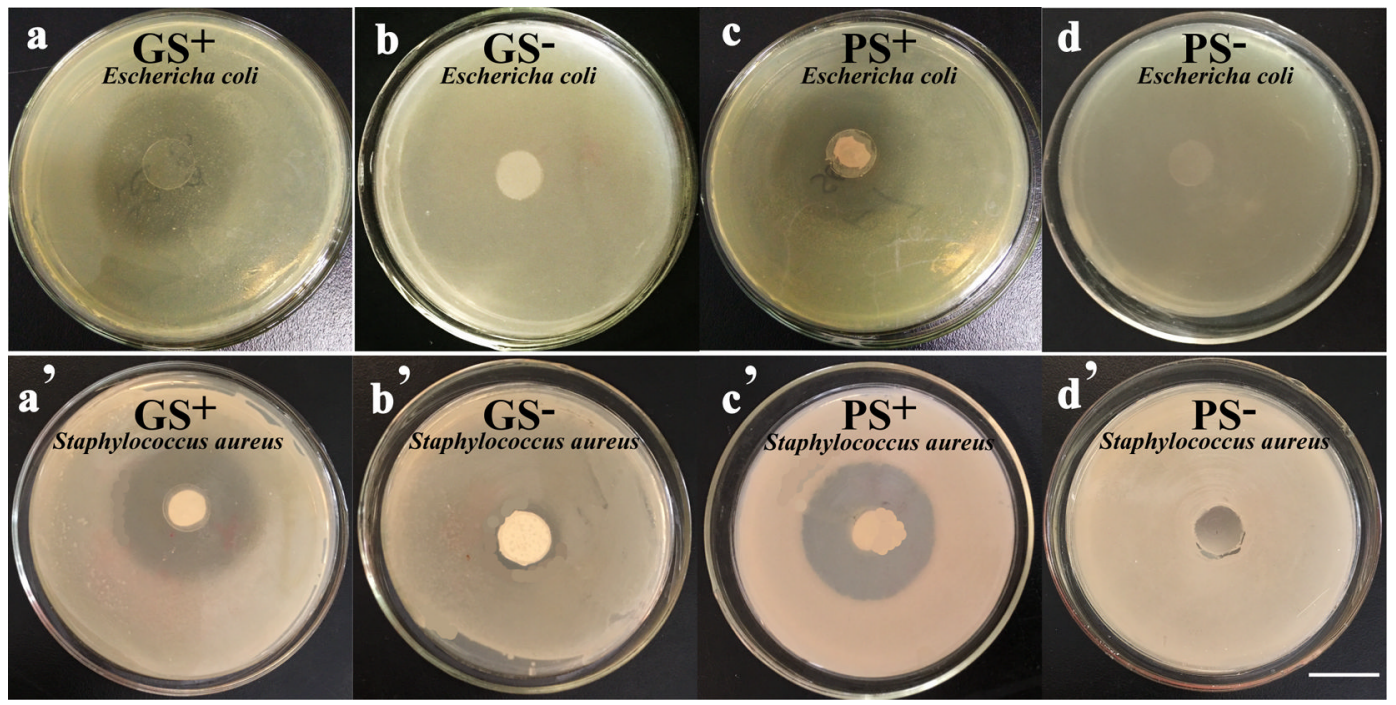

Fig. 6. The antibacterial properties of the different fibers: (a, a') GS ${ }^{+}$; (b, b') GS ; (c, c') PS ${ }^{+}$; (d, d') PS Representative images from three times experiments are shown, with inhibition zones quantified as mean \pm S.D. from all experiments. Scale bar: $20 \mathrm{~mm}$.

Table 3. Inhibition zone diameters for the fibers.

\begin{tabular}{ccc}
\hline Sample & $\begin{array}{c}\text { Inhibition zone with } \\
\text { Eschericha coli }(\mathbf{m m})\end{array}$ & $\begin{array}{c}\text { Inhibition zone with } \\
\text { Staphylococcus aureus }(\mathbf{m m})\end{array}$ \\
\hline $\mathrm{GS}^{+}$ & $36 \pm 3.7$ & $38 \pm 3.5$ \\
$\mathrm{G}^{+}$ & $35 \pm 2.5$ & $36 \pm 2.9$ \\
$\mathrm{PS}^{+}$ & $31 \pm 3.5$ & $32 \pm 1.9$ \\
$\mathrm{P}^{+}$ & $28 \pm 2.1$ & $31 \pm 3.4$ \\
$\mathrm{GS}^{-}$ & - & - \\
$\mathrm{G}^{-}$ & - & - \\
$\mathrm{PS}^{-}$ & - & - \\
$\mathrm{P}^{-}$ & - & - \\
\hline
\end{tabular}

\subsection{Biocompatibility}

The results of MTT cell viability measurements for the gelatin and PLCL nanofibers are given in Fig. 7 and Fig. S3. Evidently, the number of cells increases continuously from day 1 to day 5 in all cases. These 
results indicate that L929 cells can proliferate on both the gelatin and PLCL fibers, with no indication of any toxicity. In general, L929 fiber cells grow faster on the gelatin fibers than on the PLCL fibers, presumably because the former is a natural and hydrophilic material. The addition of ciprofloxacin did not change the biocompatibility of the fibers. Cell growth on $\mathrm{GS}^{+}$and $\mathrm{PS}^{+}$(Fig. 7) is greater than on $\mathrm{GS}^{-}$and $\mathrm{PS}^{-}$(Fig. S3). This is thought to be because SB increased the wettability of the fibers, which promotes cell growth. In all cases, the viability with the fibers is at least on a par with the untreated cells control, confirming that all the fibers have high biocompatibility.

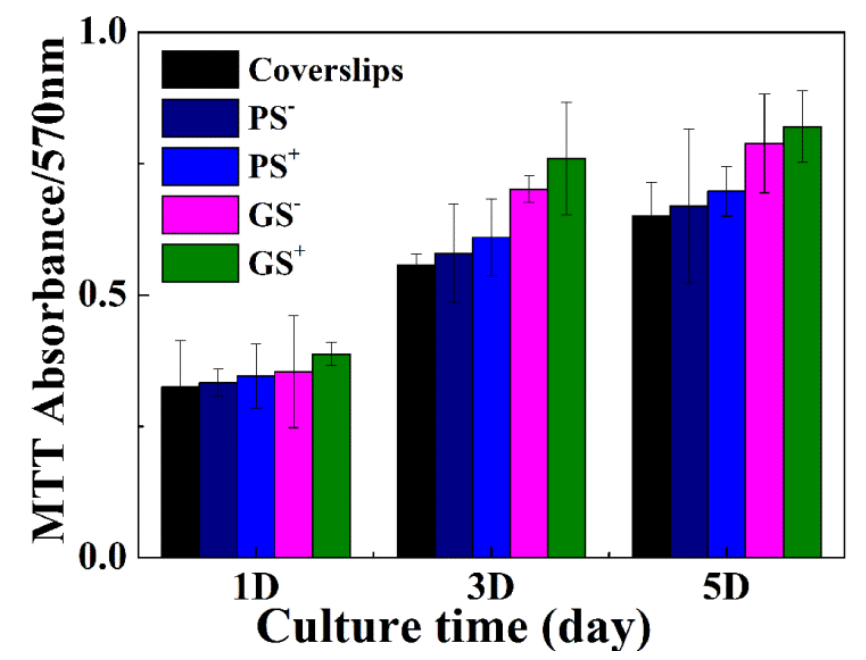

Fig. 7. The viability of L929 cells exposed to selected fibers. Data from three independent experiments are given, with 3 replicates per experiment, and are reported as mean \pm S.D.

\section{Conclusions}

A series of nanofibers were prepared comprising gelatin or PLCL doped with sodium bicarbonate (SB). Drug-loaded fibers containing the antibacterial ciprofloxacin were also produced. Electron microscopy demonstrated that all the fibers are cylindrical monoliths with smooth and uniform surfaces. IR spectroscopy verified the presence of the ciprofloxacin in the fibers, while XRD revealed the drug to be incorporated in the amorphous form. All the gelatin fibers are hydrophilic while those of PLCL are hydrophobic, but in both cases the inclusion of SB or ciprofloxacin increases the wettability. The hydrophilic gelatin-based fibers release ciprofloxacin more rapidly than the hydrophobic PLCL analogues. The gelatin/SB fibers show $\mathrm{pH}$ sensitivity, with more rapid release at lower $\mathrm{pH}$ owing to reaction of SB with protons in the acidic environment. SB-free gelatin fibers have no such sensitivity. The PLCL systems also have no $\mathrm{pH}$-sensitivity, regardless of the presence or absence of SB. All the drug-loaded fibers had antibacterial activity, and were found to be highly biocompatible with L929 cells. The gelatin fibers have greater antibacterial activities, and also biocompatibility, than the PLCL systems. Overall, the new systems prepared in this work have great potential as drug delivery systems. The $\mathrm{pH}$-sensitive properties of the gelatin/SB fibers might render them suitable as a dosage form for implantation after tumor resection, to prevent infection.

\section{Acknowledgements}

This investigation was supported by grant 16410723700 from the Science and Technology Commission of Shanghai Municipality, the Biomedical Textile Materials "111 Project" of the Ministry of Education 
of China (No. B07024), and the UK-China Joint Laboratory for Therapeutic Textiles (based at Donghua University)

\section{References}

[1] K. Dan, S.S. Liow, X. J. Loh, Biodegradable polymers for electrospinning: Towards biomedical applications, Materials Science \& Engineering C, 45 (2014) 659-670.

[2] D. Kai, M. J. Tan, M. P. Prabhakaran, B.Q.Y. Chan, S. S. Liow, S. Ramakrishna, X. J. Loh, Biocompatible electrically conductive nanofibers from inorganic-organic shape memory polymers, Colloids \& Surfaces B Biointerfaces, 148 (2016) 557-565.

[3] W. Lu, J. Sun, X. Jiang, Recent advances in electrospinning technology and biomedical applications of electrospun fibers, Journal of Materials Chemistry B, 2 (2014) 2369-2380.

[4] D. Kai, S. Jiang, Z. W. Low, X.J. Loh, Engineering highly stretchable lignin-based electrospun nanofibers for potential biomedical applications, Journal of Materials Chemistry B, 3 (2015) 6194-6204.

[5] S.H. Ranganath, C.H. Wang, Biodegradable microfiber implants delivering paclitaxel for post-surgical chemotherapy against malignant glioma, Biomaterials, 29 (2008) 2996-3003.

[6] D. Liu, S. Liu, X. Jing, X. Li, W. Li, Y. Huang, Necrosis of cervical carcinoma by dichloroacetate released from electrospun polylactide mats, Biomaterials, 33 (2012) 4362-4369.

[7] J. Hu, D. Kai, H. Ye, L. Tian, X. Ding, S. Ramakrishna, X.J. Loh, Electrospinning of poly(glycerol sebacate)based nanofibers for nerve tissue engineering, Materials Science \& Engineering C, 70 (2017) 1089-1094.

[8] M.V. Natu, H.C. de Sousa, M.H. Gil, Effects of drug solubility, state and loading on controlled release in bicomponent electrospun fibers, International Journal of Pharmaceutics, 397 (2010) 50-58.

[9] X.J. Loh, P. Peh, S. Liao, C. Sng, J. Li, Controlled drug release from biodegradable thermoresponsive physical hydrogel nanofibers, Journal of Controlled Release Official Journal of the Controlled Release Society, 143 (2010) 175-182.

[10] L.E. Aguilar, A.R. Unnithan, A. Amarjargal, A.P. Tiwari, S.T. Hong, C.H. Park, C.S. Kim, Electrospun polyurethane/Eudragit(®) L100-55 composite mats for the $\mathrm{pH}$ dependent release of paclitaxel on duodenal stent cover application, International Journal of Pharmaceutics, 478 (2015) 1-8.

[11] Z. Jingwen, L. Shen, L. Bin, Y. Huilin, F. Cunyi, C. Wenguo, Stable acid-responsive electrospun biodegradable fibers as drug carriers and cell scaffolds, Macromolecular Bioscience, 13 (2013) 885-892.

[12] P.A. Ganz, A teachable moment for oncologists: Cancer survivors, 10 million strong and growing!, Journal of Clinical Oncology, 23 (2005) 5458-5460.

[13] L. Bai, G.P. Mao, C.P. Cao, Effects of inflammatory cytokines on the recurrence of liver cancer after an apparently curative operation, Journal of Digestive Diseases, 8 (2007) 154-159.

[14] D.W. Edlow, W.H. Sheldon, The pH of inflammatory exudates, Experimental Biology and Medicine, 137 (1971) 1328-1332.

[15] K. Halake, H.J. Kim, M. Birajdar, B.S. Kim, H. Bae, C.C. Lee, Y.J. Kim, S. Kim, S. Ahn, Y.A. Su, Recently developed applications for natural hydrophilic polymers, Journal of Industrial \& Engineering Chemistry, 40 (2016) 16-22.

[16] R. Lakshminarayanan, R. Sridhar, X.J. Loh, M. Nandhakumar, V.A. Barathi, M. Kalaipriya, J.L. Kwan, S.P. Liu, R.W. Beuerman, S. Ramakrishna, Interaction of gelatin with polyenes modulates antifungal activity 
and biocompatibility of electrospun fiber mats, International Journal of Nanomedicine, 9 (2014) 2439-2458.

[17] D. Kołbuk, P. Sajkiewicz, K. Maniura-Weber, G. Fortunato, Structure and morphology of electrospun polycaprolactone/gelatine nanofibres, European Polymer Journal, 49 (2013) 2052-2061.

[18] B. Mandal, J. Mann, Sc, Silk fibroin/gelatin multilayered films as a model system for controlled drug release, European Journal of Pharmaceutical Sciences, 37 (2009) 160-171.

[19] H. Li, M. Wang, G.R. Williams, J. Wu, X. Sun, Y. Lv, L.M. Zhu, Electrospun gelatin nanofibers loaded with vitamins A and E as antibacterial wound dressing materials, Rsc Advances, 6 (2016) 50267-50277.

[20] Y.Z. Zhang, J. Venugopal, Z.M. Huang, C.T. Lim, S. Ramakrishna, Crosslinking of the electrospun gelatin nanofibers, Polymer, 47 (2006) 2911-2917.

[21] W. Fu, Z. Liu, B. Feng, R. Hu, X. He, H. Wang, M. Yin, H. Huang, H. Zhang, W. Wang, Electrospun gelatin/PCL and collagen/PLCL scaffolds for vascular tissue engineering, International Journal of Nanomedicine, 9 (2014) 2335-2344.

[22] D. Kai, M.P. Prabhakaran, B.Q. Chan, S.S. Liow, S. Ramakrishna, F. Xu, X.J. Loh, Elastic poly(عcaprolactone)-polydimethylsiloxane copolymer fibers with shape memory effect for bone tissue engineering, Biomedical Materials, 11 (2016) 015007.

[23] X.S. Pan, J. Ambler, S. Mehtar, L.M. Fisher, Involvement of topoisomerase IV and DNA gyrase as ciprofloxacin targets in Streptococcus pneumoniae, Antimicrobial Agents \& Chemotherapy, 40 (1996) 2321-2326.

[24] S. Subhashree, C.C. Kanti, B.P. Kumar, In vitro antibacterial activities study of polymeric ciprofloxacin suspensions, International Research Journal of Pharmacy, 3 (2012) 302-304.

[25] D. Kai, W. Ren, L. Tian, L.C. Pei, Y. Liu, S. Ramakrishna, J.L. Xian, Engineering Poly(lactide)-Lignin Nanofibers with Antioxidant Activity for Biomedical Application, ACS Sustainable Chemistry \& Engineering, 4 (2016) 5268-5276.

[26] L. Mafra, S.M. Santos, R. Siegel, I. Alves, F.A. Paz, D. Dudenko, H.W. Spiess, Packing interactions in hydrated and anhydrous forms of the antibiotic ciprofloxacin: a solid-state NMR, X-ray diffraction, and computer simulation study, Journal of the American Chemical Society, 134 (2011) 71-74.

[27] R. Manjula, K. Priya Dasan, M.A. Joseph, SF/poly(ethylene-co-vinyl acetate) blends for controlled drug release, International Journal of Pharmtech Research, DOI (2012) 1643-1652.

[28] H. Staroszczyk, K. Sztuka, J. Wolska, A. Wojtasz-Pająk, I. Kołodziejska, Interactions of fish gelatin and chitosan in uncrosslinked and crosslinked with EDC films: FT-IR study, Spectrochimica Acta Part A Molecular \& Biomolecular Spectroscopy, 117 (2014) 707-712.

[29] S. Kassi, K. Didriche, C. Lauzin, A. Rizopoulos, M. Herman, Demonstration of cavity enhanced FTIR spectroscopy using a femtosecond laser absorption source, Spectrochimica Acta Part A Molecular \& Biomolecular Spectroscopy, 75 (2010) 142-145.

[30] L.U. Xi-Li, L.Ü. Xiu-Qian, J.Y. Wang, Z.J. Sun, Y.X. Tong, Preparation and shape memory properties of TiO2/PLCL biodegradable polymer nanocomposites, Transactions of Nonferrous Metals Society of China, 23 (2013) 120-127.

[31] S. Pandey, P. Pandey, G. Tiwari, R. Tiwari, A.K. Rai, FTIR Spectroscopy: A tool for quantitative analysis of ciprofloxacin in tablets, Indian Journal of Pharmaceutical Sciences, 74 (2012) 86-90.

[32] X. Yang, Q. Xu, N. Yan, G. Sui, Q. Cai, X. Deng, Structure and wettability relationship of coelectrospun 
poly (L- lactic acid)/gelatin composite fibrous mats, Polymers for Advanced Technologies, 22 (2011) 22222230.

[33] Y. Zhang, H. Ouyang, C.T. Lim, S. Ramakrishna, Z.M. Huang, Electrospinning of gelatin fibers and gelatin/PCL composite fibrous scaffolds, Journal of Biomedical Materials Research Part B: Applied Biomaterials, 72B (2005) 156-165.

[34] N. Hiraishi, C.K.Y. Yiu, N.M. King, F.R. Tay, D.H. Pashley, Chlorhexidine release and water sorption characteristics of chlorhexidine-incorporated hydrophobic/hydrophilic resins, Dental Materials, 24 (2008) 1391-1399.

[35] C.J. Ke, T.Y. Su, H.L. Chen, H.L. Liu, W.L. Chiang, P.C. Chu, Y. Xia, H.W. Sung, Smart multifunctional hollow microspheres for the quick release of drugs in intracellular lysosomal compartments, Angewandte Chemie, 50 (2011) 8086-8089.

[36] N.X. Chin, H.C. Neu, Post-antibiotic suppressive effect of ciprofloxacin against gram-positive and gramnegative bacteria, American Journal of Medicine, 82 (1987) 58-62. 